\title{
Protein Detection and Localization in Plant Cells Using Spot-Tagging
}

Andriani Mentzelopoulou ${ }^{1,2 *}$, Chen $\operatorname{Liu}^{3,4^{*}}$, Panagiotis Nikolaou Moschou ${ }^{1,2,3}$

${ }^{1}$ Institute of Molecular Biology and Biotechnology, Foundation for Research and Technology - Hellas, Heraklion, Greece

${ }^{2}$ Department of Biology, University of Crete, Heraklion, Greece

${ }^{3}$ Department of Plant Biology, Uppsala BioCenter, Swedish University of Agricultural Sciences and Linnean Center for Plant Biology, Uppsala, Sweden

${ }^{4}$ Lead contact:

Chen Liu

chen.liu@slu.se

*These authors contributed equally to this work

Material Distribution:

Chen Liu chen.liu@slu.se 


\section{ABSTRACT}

2 Fluorescent labelling of proteins without compromising their activity is crucial for

3 determining their spatiotemporal localization while retaining their functionality. Spot-tag

4 is a 12-amino acid peptide recognized by a single-domain nanobody. Here we introduce

5 the spot-tag as a labelling strategy for proteins in fixed and living plant cells, using as an

6 example the microtubule motor centromeric protein E-related Kinesin 7.3. Spot-tagging of

7 ectopically introduced Kinesin 7.3 does not interfere with microtubules and spot staining

8 results in a close-grained fluorophore labelling revealing a localization pattern that

9 resembles "beads-on-a-string". We anticipate that our protocol will apply to many more

10 demanding protein cellular targets, offsetting activity perturbations and low photon

11 quantum yields imposed by other protein-tagging approaches. 


\section{INTRODUCTION}

14 Most current labelling strategies exploit antibodies or recombinant proteins fused to

15 various stable or photoactivatable fluorescent proteins (FPs) or fluorogenic-labelling

16 enzymes, e.g. the Halo-, CLIP-, or SNAP-tag (Virant et al., 2018). Conventional antibodies

17 introduce significant linkage errors by displacing the fluorophore from the target, while

18 large protein/enzyme tags may affect expression, cellular localization, folding and/or

19 function. Although small peptidic epitopes, e.g FLAG-, HA-, or Myc-tag are widely used,

20 which can be also arranged in tandem arrays to recruit medium-affine binding antibodies,

21 they may not provide sufficient labelling.

22 Instead of using antibodies, a 15-amino-acid peptide-tag can be probed by high-

23 avid fluorescently labelled monomeric streptavidin, which, however, can be affected by

24 the binding of endogenously biotinylated proteins, especially in plant cells (Arora et al.,

25 2020). Alternatively, reversibly on-/off-binding labels in point accumulation for imaging

26 of nanoscale topography (PAINT) microscopy allow for a continuous and therefore ultra-

27 high-density readout as they are not limited by a predefined fluorophore tagging pattern

28 (Schnitzbauer et al., 2017). Yet, this approach can only be used for structures like

29 membranes or DNA combined with illumination-confined arrangements, e.g. surface-near

30 or light-sheet illuminations.

31 As a promising substitute for conventional antibodies, small-sized nanobodies

32 (antibody fragments derived from heavy-chain-only camelid antibodies) coupled with

33 organic dyes, e.g. ATTO probes, were recently introduced (Virant et al., 2018). Despite

34 their capability to directly probe endogenous antigens, the de novo production of

35 nanobodies and their validation is cumbersome and time-consuming; only a very limited 
36 number of microscopy-compatible nanobodies are available by now. Due to their

37 applicability for nanoscopy of widely used FP-fusions, GFP-, and RFP-nanobodies became

38 extremely popular tools. This strategy, however, relies on the correct expression of FP-

39 fusions and does not cope with problems arising from mislocalization, steric hindrances or

40 dysfunction. Thus, nanobodies directed against short and functionally inert tags might

41 prove advantageous.

42 On par with these approaches, the versatile labelling and detection strategy

43 comprised the short and inert "spot" peptide-tag (PDRKAAVSHWQQ) and a

44 corresponding high-affinity bivalent nanobody has been successfully used in animal cells

45 (Virant et al., 2018). Here we refine the spot-tagging approach for high-resolution imaging

46 of plant cells. We demonstrate the benefits of our approach for protein labelling with

47 minimal linkage errors in fixed and living cells using the paradigmatic component of the

48 centromeric protein E-related Kinesin-Separase Complex (KISC) Kinesin 7.3 (Kin7.3).

\section{Results and Discussion}

\section{Spot-tag method establishment}

51 Kin7.3 is a core component of the KISC with predominant microtubule (MT) localization.

52 KISC modulates cell polarity and MT dynamics during plant growth. Kin7.3 is partially

53 redundant with Kin7.1 and Kin7.5, which also comprise KISC (Moschou et al., 2016).

54 Firstly, we examined the applicability of the spot tagging method by generating stable $A$.

55 thaliana transgenic lines expressing Kin7.3 fused with His/FLAG epitopes, along with N-

56 terminal spot and C-terminal myc sequences under the meristematic-specific RPS5a

57 promoter (pRPS5a::HF-spot-Kin7.3-myc). Loss-of-function kin7.1kin7.3kin7.5 (k135) 
58 mutant leaves are elongated and curly (Supplemental file, Fig. S1). We could see partial

59 complementation in plants expressing pRPS5a::HF-spot-Kin7.3-myc in the $k 135$ mutant

60 background (Supplemental file, Fig. S1). As live imaging detection of spot fluorescence

61 was only partially successful showing signal only in the outermost root cell layers

62 (Supplemental file, Fig. S2), we assumed that spot cannot be delivered successfully to inner

63 cell layers. We thus developed a protocol for spot-tagging of $A$. thaliana root tip fixed-cells

64 (Fig. 1A). We incubated fixed-roots in fluorescent anti-spot nanobodies for $1 \mathrm{~h}$ or overnight

65 and we counterstained spot-labelled roots with anti-myc (Fig. 1B). Using this approach,

66 we could detect colocalized myc/spot signal, but the intensity for both signals was rather

67 low (Fig. 1B), consistent with the reported low native expression of Kin7.3 protein

68 (Moschou et al., 2016). Taken together, these data suggest that spot-tagging is functional

69 in, at least, fixed Arabidopsis root cells.

\section{Kin7.3 detection by spot nanobodies in Nicotiana benthamiana leaves}

71 We strived to detect Kin7.3 using spot in the transient mesophyll protein expression system

72 of $N$. benthamiana. We first attempted infiltration of the spot-tag in intact tissue of $N$.

73 benthamiana leaves, which had been infiltrated with a construct carrying pro35::spot-

74 Kin7.3. Under this experimental setting, we could not detect the spot signal. Thus, spot

75 nanobodies cannot be taken up by the mesophyll cells, at least in our settings.

Next, to overcome the physical barrier of the cell wall in spot-tag uptake, we

77 introduced small wounds to the leaf tissue. To this end, we used a razor blade and re-

78 incubated the leaves using spot-tag for $1 \mathrm{~h}$. Under this setting, we could detect high-

79 intensity signal on MTs in cells which were closest to the wounds (Fig. 2A). Our results 
80 suggest that this simple wounding approach is sufficient to allow the penetration of spot

81 nanobodies into plant cells.

\section{Kin7.3 shows a "beads on a string"' localization atop cortical MTs}

83 We carried out a thorough examination of MT-based localization of Kin7.3-spot signal.

84 Subcellular localization of Kin7.3 is not affected by the spot-tagging as almost all the signal

85 resided on MTs (Fig. 2A). Surprisingly, Kin7.3-spot signal decorated unevenly the MTs

86 (Fig. 2B), in a pattern reminiscent of "beads-on-a-string" localization observed for other

87 MT- or actin-related proteins (e.g. (Buschmann et al., 2015, Wang et al., 2019)) or proteins

88 that mediate ER-plasma membrane contacts (Wang et al., 2014). On the contrary, using the

89 pro35S::GFP-Kin7.3 under the same conditions (Fig. 2C, D), we could see the increased

90 signal of GFP-Kin7.3 in the cytoplasm suggesting that GFP leads to stereochemical

91 hindrance restricting localization of Kin7.3 on MTs. Moreover, some MTs were under

92 tension perhaps due to the MT overstabilization effect of Kin7.3, leading to a spiral-like

93 MT arrangement (Fig. 2C). Furthermore, as reported previously, the MTs that were

94 decorated by Kin7.3-spot were attached to the plasma membrane, suggesting that Kin7.3

95 may be involved in plasma membrane-MTs links. Although efficient MT-binding by

96 Kin7.3 depends on the open conformation of Kin7.3 were N- and C-termini of Kin7.3 are

97 far apart due to C-terminal binding of separase, the spot-nanobody may function in the

98 same way by blocking the intramolecular N/C-interaction of Kin7.3 allowing increased

99 MT-binding (model in Fig. 2E).

100 Conclusion 
101 We foresee that our spot-tagging approach of can be widely applicable in plant biology,

102 while the two available conjugated to spot fluorophores (i.e. ATTO488/561) will allow

103 detection in at least two different emission wavelengths easing colocalization studies. The

104 high quantum yield of spot-conjugated ATTO probes and their photostability may allow

105 gaining detailed and high-resolution images of spot-tagged proteins, for which fluorescent-

106 images cannot be easily obtained due to low expression levels and/or photostability or

107 quantum yields. Hence, the spot-tagging approach may enable single-molecule localization

108 microscopy (SMLM) techniques, providing outstanding spatial resolutions surpassing

109 limitations, such as poor photon emission or detection efficiency, low fluorophore labelling

110 densities, linkage errors or steric hindrances.

111 Materials and Methods

\section{Plant material and growth conditions}

113 Arabidopsis thaliana Col-0 plants were grown on vertical square plates containing half114 strength Murashige and Skoog medium (Duchefa), supplemented with 1\% (w/v) sucrose 115 and $0.7 \%(\mathrm{w} / \mathrm{v})$ plant agar. Nicotiana benthamiana plants were, directly, grown on soil.

116 Plant growth was done at $22^{\circ} \mathrm{C}$ on a 16-hr/8-hr light/dark cycle and light intensity of 150 $117 \mu \mathrm{E} \mathrm{m}^{-2} \mathrm{~s}^{-1}$ in a photostable growth chamber (FitoClima 6001.200; Aralab). Arabidopsis

118 plants were transformed according to Clough and Bent (Clough \& Bent, 1998) using 119 Agrobacterium tumefaciens strain GV3101 carrying pRPS5a::HF-spot-Kin7.3-myc 120 construct in a pGWB-based vector (Nakagawa et al., 2007). 


\section{Molecular Biology and Vectors Construction}

122 Kin7.3 coding sequence was amplified from Arabidopsis thaliana first-strand cDNA

123 template via RT-PCR. The PCR amplification product was cloned in pDONR221 and

124 subsequently in destination Gateway vectors by BP and LR Gateway recombination 125 reactions, respectively. Sequences of the primers used in this study are available in

126 Supplemental Table 1. The spot tag sequence introduced was PDRVRAVSHWSS.

\section{Agroinfiltration}

Agrobacterium tumefaciens GV3101 strain, carrying pro35S::Kin7.3-spot were cultured at $28^{\circ} \mathrm{C}$ in Yeast Extract Peptone (YEP) liquid medium (1\% (w/v) yeast extract, $1 \%(\mathrm{w} / \mathrm{v})$ peptone, $0.5 \%(\mathrm{w} / \mathrm{v}) \mathrm{NaCl}$ ) supplemented with $50 \mathrm{ug} / \mathrm{ml}$ Spectinomycin and $50 \mathrm{ug} / \mathrm{ml}$

131 Rifampicin. Bacteria were harvested by centrifugation at 2,800 $\mathrm{g}$ for $10 \mathrm{~min}$ and the pellet 132 was re-suspended in buffer containing $10 \mathrm{mM}$ MES pH 5.7, $10 \mathrm{mM} \mathrm{MgCl}_{2}$ and 200uM acetosyringone; the bacteria were left agitating for $2-4$ hours at $28^{\circ} \mathrm{C}$. Agrobacteria were

134 harvested, again, respectively, and infiltrated into mesophyll leaves of $N$. benthamiana at 135 a final $\mathrm{OD}_{600}$ of 0.4 , while co-expressed with p19 silencing suppressor. Approximately six136 week-old $N$. benthamiana leaves were used for the infiltration assay.

\section{Spot tag detection}

138 Roots from A. thaliana pRPS5a::HF-spot-Kin7.3-myc transgenic lines were fixed in 139 fixation buffer for $1 \mathrm{~h}\left(3.7 \%\right.$ paraformaldehyde (PFA; dissolved in warm $\mathrm{ddH}_{2} \mathrm{O}$ 140 supplemented with 2 drops of $0.1 \mathrm{~N} \mathrm{KOH}$ ), 50mM piperazine-N,N'-bis (2-ethane sulfonic 141 acid) (PIPES), $5 \mathrm{mM}$ Ethylene glycol tetraacetic acid (EGTA), $2 \mathrm{mM} \mathrm{MgSO} 4,0.4 \%$ (v/v) 142 Triton X-100). Roots were separated from the seedlings using a razor blade and washed 
143 with Phosphate-buffered saline/0.01\% (v/v) Tween-20 (PBST) buffer and next, they were

144 treated with driselase enzyme buffer for $7 \mathrm{~min}(2 \%(\mathrm{w} / \mathrm{v})$ Driselase (Sigma), $0.4 \mathrm{M}$

145 Mannitol, 5mM EGTA, 15mM 2-(N-morpholino) ethane sulfonic acid (MES), pH 5.6, 1

$146 \mathrm{mM}$ phenylmethylsulfonyl fluoride protease inhibitor (PMSF; Sigma)). Epitope blocking

147 was done for $30 \mathrm{~min}$ at RT using 3\% (w/v) BSA/PBST. Then, roots were incubated

148 overnight at $4{ }^{\circ} \mathrm{C}$ in anti-myc-mouse primary antibody (1:250, Roche in blocking solution),

149 washed 3 times in PBST. The roots were incubated overnight at $4^{\circ} \mathrm{C}$ with the secondary

150 anti-mouse (1:500; Jackson immunoreactive, conjugated to Rhodamine Red) and eba488-

15110 Spot Label ATTO488 (1:1000, Chromotek) diluted in PBS supplemented with 2\% (w/v)

152 BSA. After washing in PBST buffer supplemented with DAPI (4',6-diamidino-2-

153 phenylindole), roots were mounted in Vectashield (Vector Laboratories) mounting 154 medium.

$N$. benthamiana leaves were harvested 3 days post infiltration with the 156 corresponding Agrobacteria carrying the constructs of interest (i.e. spot-Kin7.3 or GFP157 Kin7.3). Small cuts from the infiltrated regions were incubated for one hour in anti-Spot158 Tag VHH/Nanobodies conjugated to the organic fluorophore ATTO488 (Chromotek), 159 diluted 1:1000 in $10 \mathrm{mM}$ Tris- $\mathrm{HCl}$ solution $\mathrm{pH}$ 7.5, adjusted with $\mathrm{HCl}$.

160 The fluorescent images (micrographs) were acquired at RT using the Leica SP8 161 inverted confocal microscope, with 40x objective (N.A.=1.2), pinhole adjusted to 1 airy 162 unit, and mounting medium $\mathrm{ddH}_{2} \mathrm{O}$. The excitation wavelength was $488 \mathrm{~nm}$ (Emission $163506 \mathrm{~nm}-544 \mathrm{~nm})$ for the spot tag detection and $552 \mathrm{~nm}$ (Emission 562nm - 636nm) for anti164 myc/Rhodamine detection and 405nm (Emission 412nm - 467nm) for DAPI (nuclei 165 detection). The excitation wavelength for chlorophyll was $488 \mathrm{~nm}$ (Emission 764nm - 
166

167

168

769nm). For fixed-cell imaging, we used 40x oil corrected objective (N.A.=1.3; reflective index n20/D 1.516).

\section{Image analysis}

The image and pixel analyses were done using IMAGEJ software (https://imagej.nih.gov/ij/). Plot profile was calculated along an interactively applied line, and data of intensity measurements were exported to Microsoft EXCEL (Microsoft, Redmond, WA, USA) and plotted. Default modules and options were used. Images were prepared using Adobe PHOTOSHOP (Adobe, San Jose, CA, USA).

\section{FIGURE LEGENDS}

Figure 1. Spot-tag experimental pipeline in stable $A$. thaliana transgenic lines carrying pRPS5a::HF-spot-Kin7.3-myc.

A. Experimental pipeline for spot detection in fixed root meristematic cells. Scale, 10 um.

B. Detection of spot-tag and myc/Rhodamine signal in root epidermis meristematic cells and colocalization analysis using plot profile. Pixel colocalization analysis using Pearson correlation coefficient was $\sim+0.5$.

Figure 2. Transient expression and detection of spot-kin7.3 in $N$. benthamiana mesophyll cells.

A. Spot-tag detection of spot-Kin7.3 expressed in wounded but alive $N$. benthamiana mesophyll cells.

B. "Beads-on-a-string" detection of spot-Kin7.3 signal (denoted with white arrowheads).

C. Spiral arrangement of MTs imposed by tension due to overstabilization effect and/or overaccumulation of spot-Kin7.3.

D. Detection of GFP-Kin7.3 expressed under 35S promoter. Note the increased cytoplasmic signal. 
E. Model of Kin7.3 activation and binding of MTs. In the absence of Kin7.3 or spot nanobodies, Kin7.3 is in the closed conformation (upper). Separase finds on the non-motor C-terminus tail domain, thereby blocking N/C-termini interaction (middle). Spot nanobodies bind on the N-terminus of spot-Kin7.3 leading to a sterical hindrance that allows Kin7.3 binding on MTs.

\section{ACKNOWLEDGEMENTS}

Part of this work was funded by the start-up grants from IMBB-FORTH.

\section{REFERENCES}

Arora D, Abel NB, Liu C, et al., 2020. Establishment of Proximity-dependent Biotinylation Approaches in Different Plant Model Systems. The Plant Cell, tpc.00235.2020.

Buschmann H, Dols J, Kopischke S, et al., 2015. Arabidopsis KCBP interacts with AIR9 but stays in the cortical division zone throughout mitosis via its MyTH4-FERM domain. Journal of Cell Science 128, 2033-46.

Clough, S. J., \& Bent, A. F. (1998). Floral dip: A simplified method for Agrobacteriummediated transformation of Arabidopsis thaliana. Plant Journal, 16(6), 735-743. https://doi.org/10.1046/j.1365-313X.1998.00343.x

Moschou PN, Gutierrez-Beltran E, Bozhkov PV, Smertenko A, 2016. Separase Promotes Microtubule Polymerization by Activating CENP-E-Related Kinesin Kin7. Dev Cell 37, 35061.

Nakagawa T, Kurose T, Hino T, et al., 2007. Development of series of gateway binary vectors, pGWBs, for realizing efficient construction of fusion genes for plant transformation. Journal of Bioscience and Bioengineering 104, 34-41.

Schnitzbauer J, Strauss MT, Schlichthaerle T, Schueder F, Jungmann R, 2017. Superresolution microscopy with DNA-PAINT. Nat Protoc 12, 1198-228.

Virant D, Traenkle B, Maier J, et al., 2018. A peptide tag-specific nanobody enables highquality labeling for dSTORM imaging. Nature Communications 9, 930. 
bioRxiv preprint doi: https://doi.org/10.1101/2020.11.24.396507; this version posted November 24,2020 . The copyright holder for this preprint (which was not certified by peer review) is the author/funder, who has granted bioRxiv a license to display the preprint in perpetuity. It is made available under aCC-BY-NC-ND 4.0 International license.

219 Wang P, Hawkins TJ, Richardson C, et al., 2014. The plant cytoskeleton, NET3C, and VAP27

220 mediate the link between the plasma membrane and endoplasmic reticulum. Curr Biol

$22124,1397-405$.

222 Wang P, Pleskot R, Zang J, et al., 2019. Plant AtEH/Pan1 proteins drive autophagosome

223 formation at ER-PM contact sites with actin and endocytic machinery. Nature

224 Communications 10, 5132. 
A

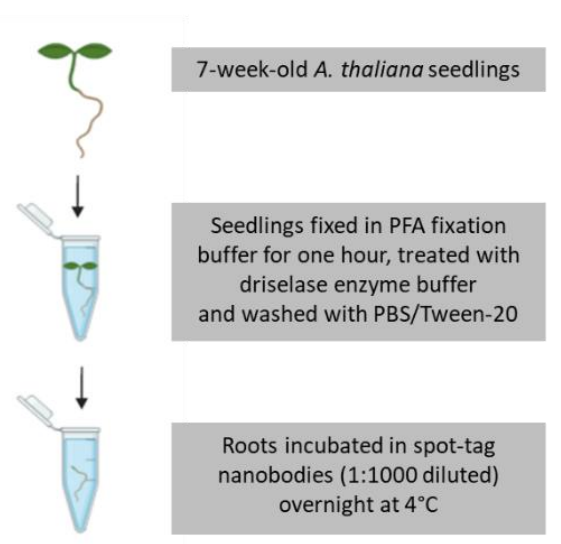

226

227

228

229

230

231

232

233 carrying pRPS5a::HF-spot-Kin7.3-myc. um.
B
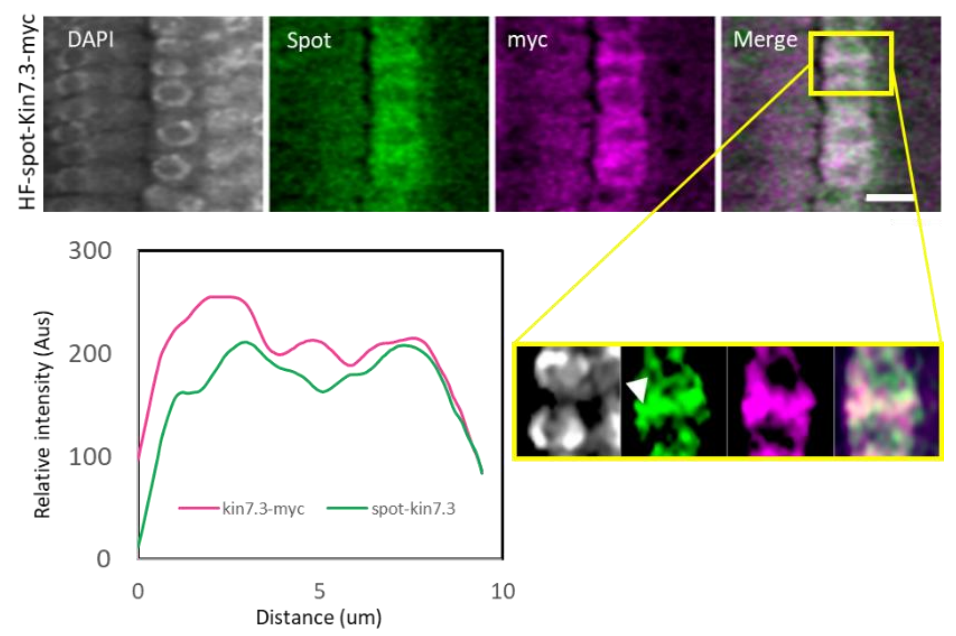

Figure 1. Spot-tag experimental pipeline in stable $A$. thaliana transgenic lines

A. Experimental pipeline for spot detection in fixed root meristematic cells. Scale, 10

B. Detection of spot-tag and myc/Rhodamine signal in root epidermis meristematic cells and colocalization analysis using plot profile. Pixel colocalization analysis using Pearson correlation coefficient was $\sim+0.5$. 

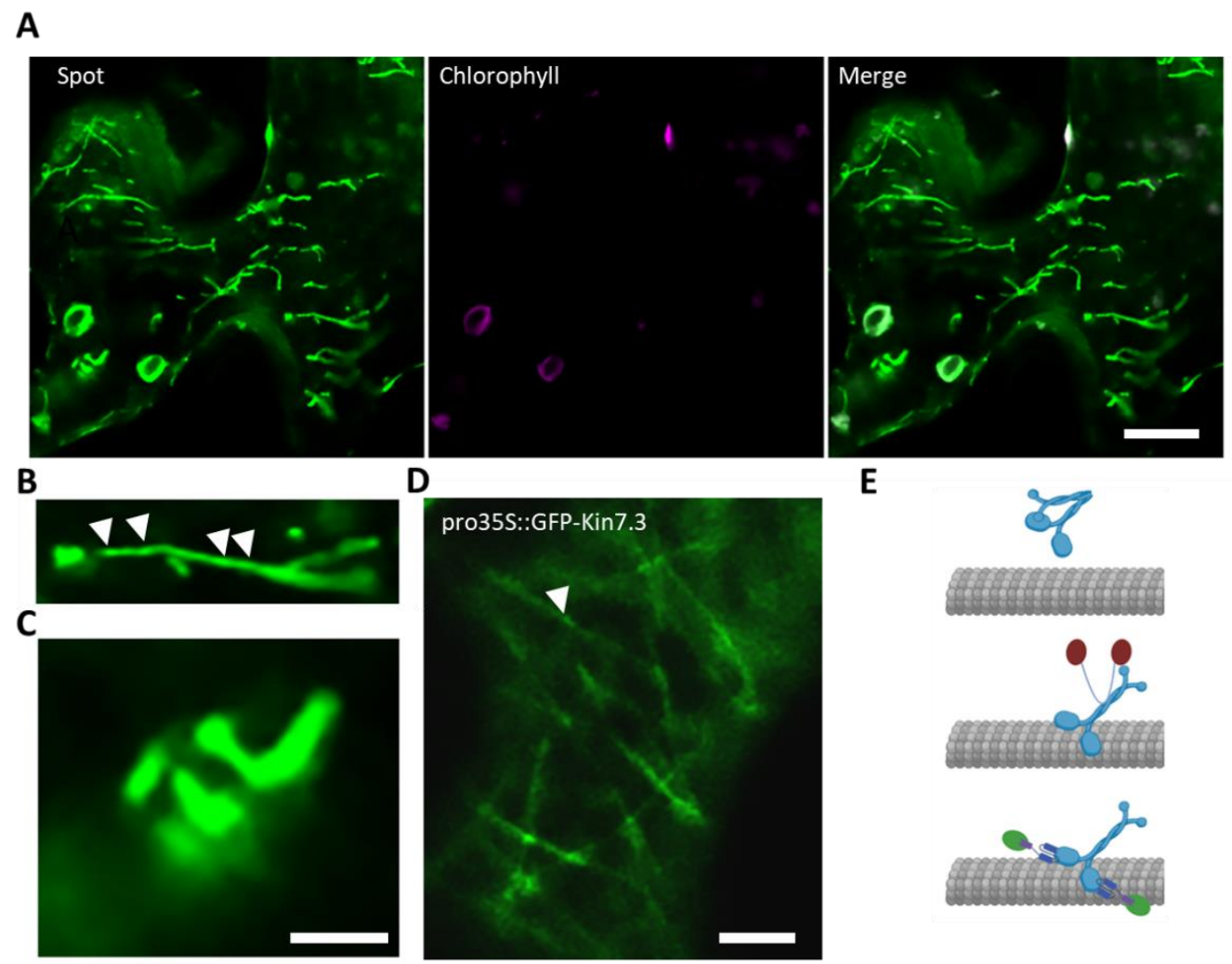

234

235

236

237

238

239

240

241

242

243

244

245

246

247

248

249

250

251

Figure 2. Transient expression and detection of spot-kin7.3 in N. benthamiana mesophyll cells.

A. Spot-tag detection of spot-Kin7.3 expressed in wounded but alive N. benthamiana mesophyll cells.

B. "Beads-on-a-string" detection of spot-Kin7.3 signal (denoted with white arrowheads).

C. Spiral arrangement of MTs imposed by tension due to overstabilization effect and/or overaccumulation of spot-Kin7.3.

D. Detection of GFP-Kin7.3 expressed under 35S promoter. Note the increased cytoplasmic signal.

E. Model of Kin7.3 activation and binding of MTs. In the absence of Kin7.3 or spot nanobodies, Kin7.3 is in the closed conformation (upper). Separase finds on the non-motor C-terminus tail domain, thereby blocking N/C-termini interaction (middle). Spot nanobodies bind on the $\mathrm{N}$-terminus of spot-Kin7.3 leading to a sterical hindrance that allows Kin7.3 binding on MTs (Created with BioRender.com). 